\title{
An Evolutionary Grey, Hop, Skip, and Jump Approach: Generating Alternative Policies for the Expansion of Waste Management Facilities
}

\author{
J. S. Yeomans ${ }^{1 *}$ and G. H. Huang ${ }^{2}$ \\ ${ }^{1}$ Management Science Area, Schulich School of Business, York University, Toronto, ON M3J 1P3, Canada \\ ${ }^{2}$ Faculty of Engineering, University of Regina, Regina, SK S4S 0A2, Canada
}

\begin{abstract}
Evolutionary simulation-optimization methods are combined with a Grey Hop, Skip, and Jump (GHSJ) approach in an application to municipal solid waste management planning. GHSJ techniques have been effectively applied to problems containing uncertain information. Simulation-optimization methods can be adapted to a wide variety of problem types in which some or all of the system components are stochastic. In this paper, the advantages from both of these techniques are combined and used for efficiently generating improved decision alternatives. An illustrative application of the method is provided to demonstrate the usefulness of this approach in the planning design phase for the expansion of a waste management system. By using this approach, multiple different planning alternatives can be created that meet established system criteria, while simultaneously remaining acceptable and implementable in practice. Solid waste decision makers faced with difficult and controversial choices would then interpret and analyze these alternatives to internalize the environmental-economic tradeoffs prior to selecting their final policy.
\end{abstract}

Keywords: Public sector decision making under uncertainty, simulation, evolutionary algorithms, waste management planning, modeling to generate alternatives

\section{Introduction}

Municipal solid waste (MSW) management systems possess many social, economic, technological, environmental, and political dimensions. Due to the inherent incompatibility of these dimensions, the difficulties in policy planning for managing and expanding MSW systems generally become convoluted through multiple stakeholders proffering conflicting agendas. Notwithstanding the lack of consensus regarding the best planning approach to pursue, Openshaw and Whitehead (1975) and Harris (1983) established the need for a formalized approach to planning, particularly for the effective design and evaluation of policies. Mathematical programming provided one such avenue for formalized planning. Optimization modeling has been extensively used for analyzing impacts of many disparate conflicting effects on MSW planning problems (Haynes, 1981; Wenger and Cruz, 1990; Marks and Liebman, 1971; Walker, 1976; Hasit and Warner, 1981; Lund, 1990; Lund et al., 1994) and for generating desirable solutions for particular problem instances. However, optimization approaches, alone, are unlikely to produce the single best policy solution that simultaneously satisfies all of the multiple conflicting dimensions identified above (Huang et al., 1996b; Rubenstein-Montano and Zandi, 1999). Yet planning models can still prove effective in facilitating the movement toward

\footnotetext{
* Corresponding author: syeomans@schulich.yorku.ca
}

the realization of objectives set by policy makers (Keeney and Raiffa, 1976; Kleindorfer et al., 1993), when it is recognized that the modeling methods used for policy setting must also be capable of incorporating direct inputs and expertise from decision makers (Rubenstein-Montano et al. 2000). Hence, as in any policy formulation context, MSW planners must balance and integrate many factors prior to the establishment of their final policy selection.

Due to the presence of considerable system uncertainty and the possibility that opposition from any major stakeholder could eliminate outright an 'optimal' solution from further consideration, MSW planners faced with difficult and controversial system expansion choices would generally prefer a set of alternatives in order to incorporate their own implicit knowledge within the resolution of the problem under analysis (Huang et al., 1996b). Preferably all such alternatives would be close to optimal when measured by their objective functions, but differ significantly from each other in terms of the system structure as characterized by their decision variables. Using both experience and intuition regarding the specific application, decision makers would then review these disparate alternatives to internalize the tradeoffs between the differences in the objective function values and the differing system structure characteristics suggested by each decision variable solution.

In response to this solution option requirement, several methods for modeling to generate alternatives (MGA) have been proposed (Baetz et al., 1990; Brill, 1979; Brill et al., 1981; Chang et al., 1980; Chang et al.1982; Church 
and Huber, 1979; Falkenhausen, 1979; Gidley and Bari, 1986; Rubenstein-Montano and Zandi, 1999; Rubenstein-Montano et al. 2000). MGA approaches provide an optimal solution and several near-optimal alternatives for planning problems (Gidley and Bari, 1986). Policy makers can then undertake a subsequent comprehensive evaluation of these planning alternatives to determine which option most appropriately satisfies their specific policy situation.

The major drawback to these MGA approaches arises from the fact that they have all been based upon deterministic mathematical programming methods and consequently cannot provide an effective means to integrate the plethora of uncertain components prevalent within all MSW sub-systems directly into their solution construction. To counteract this deficiency in processing system uncertainty, Huang et al. (1996b) combined grey mathematical programming (GP) with the deterministic Hop, Skip, and Jump (HSJ) MGA technique of Brill (1979), thereby permitting inherent system uncertainty to be directly incorporated within the policy construction and decision-making phase of the planning process. This hybrid procedure was referred to as the Grey, Hop, Skip and Jump (GHSJ) method. Huang et al. (1996b) demonstrated the utility of GHSJ modeling in generating policy alternatives by applying the method to a realistic case example of MSW expansion planning using representative cost and technical data taken from the solid waste management literature.

\section{Situation}

In HSJ modeling, after optimizing an initial problem formulation, supplementary problem instances are systematically solved with additional target constraints being placed upon both the objective function value and the decision variables which force the production of alternate solutions (Brill, 1979; Brill et al., 1981). Practice normally dictates that good alternative solutions should never be more than $10 \%$ worse than the initial problem formulation's optimal solution when additional unmodelled planning issues are included (Chang et al., 1982; Huang et al., 1996b). Hence, the supplementary options created in HSJ involve a "stepping" through several solution alternatives that are created by the inclusion of progressively incremented target constraints on the original optimal objective function sequentially inflating it by up to $10 \%$. HSJ had been designed and applied only to problems in which the model parameters were deterministic (Brill, 1979; Brill et al., 1981).

On the other hand, GP provides an approach which readily handles estimated uncertain input data expressed in the form of intervals. Interval estimation is particularly meaningful in practical situations, since it is generally far easier for practitioners to define fluctuation intervals than to specify appropriate distribution information. For MSW systems, examples of such interval uncertainty might have arisen from statements expressed in the form: "the capital cost for expanding the composting facility will be in the range of $\$ 1,000,000$ to $\$ 1,200,000$, "the waste generation rate is approximately 90 to 100 tonnes per week", "the incinerator has a capacity to process 2,000 to 2,500 tonnes of waste per week", and so forth (Huang et al., 1996b; Inuiguchi et al., 1990). In GP, a given problem with interval parameters is transformed into two deterministic submodels which, when solved in tandem, will guarantee stable upper and lower limits for the desired objective-function values. Unlike "normal" interval and best case-worst case analysis, however, the transformation must be performed in a specifically prescribed order using an interactive algorithm that incorporates the output from the first submodel as input into the solution of the second submodel. The solution output from GP is a set of stable interval values for all of the decision variables and for the objective function. GP has been used extensively for solving a number of environmental management and planning problems operating under uncertain conditions (Bass et al., 1997; Chang and Wang, 1995; Chang et al., 1996; Huang, 1994, 1996; Huang et al., 1994ab, 1995, 1996ab, 1997, 1998). In this paper, notation $[a, b]$ will be used to indicate that the value of an uncertain, or grey parameter, is estimated to lie within the numerical interval between the values of $a$ and $b$. Furthermore, for mathematical convenience, if variable $A$ is used to represent the grey interval [ $a$, $b]$, then the uncertainty surrounding this variable will be expressed using notation $\vec{A}$.

In GHSJ, a set of alternative system solutions is produced by solving a sequence of GP problems that are created by progressively adding system target constraints in a format adopted from the HSJ modeling technique. Without the need for complicated intermediate modeling, the GP aspects of GHSJ produce significant computational advantages over other MGA approaches. The major contribution of GHSJ over all of the other MGA approaches derives from its facility for incorporating inherent system uncertainties directly into the generation of the solution alternatives. The efficacy of GHSJ was demonstrated in Huang et al. (1996b) via an application to a realistic, hypothetical case study of MSW management planning.

\section{Problem Statement}

However, one significant shortcoming of the GHSJ (and of the HSJ) method arises from the fact that only a finite number of alternatives can ever be realistically created and examined by a planner, while the number of potentially feasible alternatives could prove to be extremely numerous. Hence, the restriction of the search for alternatives to only the very small subset of possibilities that might have occurred to the planner in the "stepping" phase of GHSJ, leads to the significant likelihood that many potentially better design alternatives could have been overlooked entirely. Therefore, to circumvent these myopic search tendencies, it would be advantageous to determine if a formalized process for efficiently creating 
multiple good solution alternatives could be constructed that simultaneously maintained the desirable capability for integrating inherent planning uncertainty directly into the generation of these alternatives.

\section{Goals, Targets and Hypotheses}

Yeomans (2002) recently described an evolutionary simulation-optimization procedure which provided a formal mechanism for "automatically" generating many desirable policy options that would not have been considered otherwise. However, since evolutionary procedures employ probabilistic search processes, both their solution times and solution quality are stochastic and can vary considerably from one implementation to the next (Lacksonen 2001). Consequently, although evolutionary simulation-optimization procedures can often produce impressive results, they cannot be considered an overarching panacea for addressing large complex problems due to the unpredictability introduced by these stochastic tendencies. In this paper, the computational efficiencies of the GHSJ procedure will be combined with the policy generation process of Yeomans (2002) in order to determine whether or not the solution time and performance quality of both of these methods can be improved upon by such integration. The effectiveness of this combined approach will be tested using the case study for MSW expansion planning presented in Huang et al. (1996b).

\section{Materials and Methods}

Simulation-optimization refers to a family of solution techniques that have been used for modeling complex problems possessing many stochastic and highly non-linear components in which precise analytical formulations of the investigated systems do not often exist (Fu, 1994). Many of these systems are characterized by the presence of multiple conflicting objectives (Teleb and Azadivar, 1994), while simultaneously possessing decision variables from the real, integer, qualitative and rule-based domains (Pierreval and Tautou, 1997; Azadivar and Tompkins, 1999). Generally, some or all of the objective functions and constraints are both stochastic and implicit functions of decision variables that can only be evaluated efficiently through computer simulation. Finding solutions to such problems tends to be difficult and generally necessitates the combination of simulation with an optimization-based search technique. The underlying rationale for the optimization component is to efficiently guide the exploration strategy through the solution space using only a limited number of simulation experiments (Lacksonen 2001).

In simulation-optimization, analytical objective functions and constraints are replaced by one or more discrete event simulation models in which the decision variables provide the conditions under which each simulation is run. The performance measures for a solution become one, or a function of several, of the responses generated during the simulation. The measured values determined in the simulation phase are then returned to the optimization procedure to direct the next decision variable settings and the subsequent step in the solution search process. Several researchers have employed evolutionary algorithms to guide the search optimization phase (Azadivar and Tompkins, 1999; Fontanili et al. 2000; Pierreval and Tautou, 1997; Huang et al. 2001). It should be noted that simulation-optimization has been employed strictly for function optimization purposes in all of these applications.

Yeomans (2002), however, recognized that evolutionary algorithms maintain a set of several candidate solutions (their population) throughout their searching phase, with the search progressing from one population of solutions to the next. Each candidate solution retained in a population corresponds to one possible solution option and, therefore, the entire population of these candidate solutions would represent a set of alternative solutions for a problem under study. All surviving solutions from the evolutionary actions of the optimization phase would have been retained on a "survival of the fittest" basis and the final population would necessarily be representative of a highly "fit" population. Hence, upon termination, an evolutionary process would not only have found the single best answer from its solution search, but also created a set of several "near-best" solutions residing in its terminal population. Thus, the procedure would have efficiently produced a family of several good solution alternatives for the problem studied. It is this final, "fit" population that would correspond to the set of good alternative options to be considered for implementation by policy planners. Because an evolutionary procedure has guided the solution search, these policy alternatives would have been generated "automatically". Most of these alternatives would not have been considered by the planners during a "normal" policy design phase, and all of these policy solutions would have incorporated uncertain conditions directly within their construction phase. Therefore, the evolutionary simulation-optimization process would have automatically created several good policy alternatives in the planning design phase, while concurrently incorporating stochastic system elements directly within the alternative generation process. As with all MGA methods, the planners would subsequently be required to prioritize the trade-offs between these different alternatives to determine exactly which policy would best satisfy their particular planning situation.

Unfortunately, evolutionary procedures follow a probabilistic search process when evolving from one population to a subsequent one. Hence, the actual solutions found and the time to find them are stochastic, varying considerably from one implementation of the method to the next. Furthermore, in complex systems, solution quality can be highly variable. One aspect to evolutionary simulation-optimization methods, therefore, is the determination of an initial population from which the search procedure commences. Traditionally, initial populations of candidate solutions have been randomly 
generated (Goldberg, 1989,, 1991; Holland, 1992). However, other research has indicated that a directed generation of the initial population may prove more efficient than this traditional random approach (Reeves, 1993). In this paper, the efficient solution procedure of Huang et al. (1996b) will be used to provide the starting point for the policy generation process of Yeomans (2002) in order to improve both solution time and performance quality of evolutionary simulation-optimization, while readily exploiting all of the benefits attributable to the GHSJ procedure.

\section{Mathematical Model for MSW Management Planning}

This section will provide a synopsis of the MSW case study of Huang et al. (1996b), while more extensive details concerning the source data can be found in the originating paper. The region studied includes three municipalities whose MSW disposal requirements are served through two waste-to-energy (WTE) facilities and a landfill. The physical layout of this region is shown in Figure 1. At the start of the planning period, WTE facilities 1 and 2 have processing capacities of $[100,125]$ and [200, 250] tonnes/day, respectively, and the landfill possesses an existing capacity of $[0.625,0.775] \times 10^{6}$ tonnes.

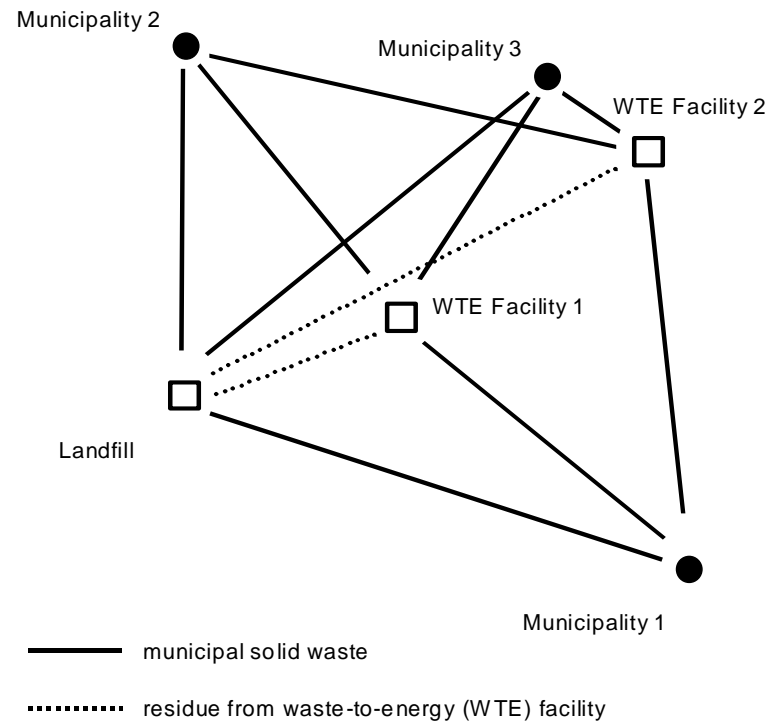

Figure 1. Location of municipalities and waste management facilities.

The WTE facilities generate residues of approximately $30 \%$ of the incoming waste streams on a mass basis, and revenue from the resulting energy re-sale is approximately $[15,25] \$ /$ tonne of combusted material.

Table 1. Capacity Expansion Options and Their Costs for the Landfill and WTE Facilities

\begin{tabular}{|c|c|c|c|}
\hline & \multicolumn{3}{|c|}{ Time Period } \\
\hline & $k=1$ & $k=2$ & $k=3$ \\
\hline \multicolumn{4}{|c|}{ Capacity expansion option for WTE facility $i, i=2,3$ (tonnes/day): } \\
\hline$\vec{\Delta} T C_{i 1 k}$ (option 1) & 100 & 100 & 100 \\
\hline$\vec{\Delta} T C_{i 2 k}$ (option 2) & 150 & 150 & 150 \\
\hline$\vec{\Delta} T C_{i 3 k}$ (option 3) & 200 & 200 & 200 \\
\hline$\vec{\Delta} T C_{i 4 k}$ (option 4) & 250 & 250 & 250 \\
\hline \multicolumn{4}{|c|}{ Capacity expansion option for the landfill ( $10^{6}$ tonnes): } \\
\hline$\vec{\Delta} L C_{k}$ & {$[1.55,1.70]$} & {$[1.55,1.70]$} & {$[1.55,1.70]$} \\
\hline \multicolumn{4}{|c|}{ Capital cost of WTE facility expansion, $i=2,3\left(\$ 10^{6}\right.$ present value): } \\
\hline$\vec{F} T C_{i 1 k}$ (option 1) & 10.5 & 8.3 & 6.5 \\
\hline$\vec{F} T C_{i 2 k}$ (option 2) & 15.2 & 11.9 & 9.3 \\
\hline$\vec{F} T C_{i 3 k}$ (option 3) & 19.8 & 15.5 & 12.2 \\
\hline$\vec{F} T C_{i 4 k}$ (option 4) & 24.4 & 19.1 & 15.0 \\
\hline \multicolumn{4}{|c|}{ Capital cost of landfill expansion $\left(\$ 10^{6}\right.$ present value $)$ : } \\
\hline$\vec{F} T C_{k}$ & {$[13,15]$} & {$[13,15]$} & {$[13,15]$} \\
\hline
\end{tabular}


Three time periods are considered with each period consisting of an interval of five years. Over the entire 15 year planning horizon, the landfill capacity can be expanded only once by an increment of $[1.55,1.70] \times 10^{6}$ tonnes. Each of the WTE facilities can be expanded by any one of four options in each of the three time periods (see Table 1 for detailed information). The maximum possible expansion option that could occur in any single time period would increase the processing capacity in a WTE facility by 250 tonnes/day. Table 1 also provides the capital costs, expressed in present value dollars, for the potential capacity expansions of the three facilities. These expansion costs escalate over time in order to reflect anticipated future conditions and have been discounted to present value cost terms for use in the objective function. The MSW waste generation rates and the costs for waste transportation and treatment vary both temporally and spatially. Table 2 provides the waste generation rates for the three municipalities, the operating costs for the three processing facilities, and the transportation costs for waste flows between municipalities and processing facilities over each of the three time periods.

Hence, the case problem requires the determination of the preferred facility expansion alternatives during the different time periods and the effective allocation of the relevant waste flows in order to minimize the total system costs over the planning horizon. In the subsequent mathematical representation of the case, the type of waste management facility will be identified using subscript $i$, with $i=1$ corresponding to the landfill, and $i=2$ and $i=3$ representing WTE facilities 2 and 3, respectively. The three municipalities from which the waste originates will be identified using subscript $j, j=1,2,3$. Subscript $k, k=$

Table 2. Waste Generation, Transportation Costs, and Facility Operating Costs

\begin{tabular}{|c|c|c|c|}
\hline & $k=1$ & $\begin{array}{c}\text { Time Period } \\
\qquad k=2\end{array}$ & $k=3$ \\
\hline \multicolumn{4}{|l|}{ Waste generation (tonnes/day): } \\
\hline$\vec{W} G_{1 k}$ (Municipality 1$)$ & {$[200,250]$} & {$[225,275]$} & {$[250,300]$} \\
\hline$\vec{W} G_{2 k}$ (Municipality 2) & {$[375,425]$} & {$[425,475]$} & {$[475,525]$} \\
\hline$\vec{W} G_{3 k}$ (Municipality 3 ) & {$[300,350]$} & {$[325,375]$} & {$[375,425]$} \\
\hline \multicolumn{4}{|c|}{ Cost of waste transportation to the landfill (\$/tonne): } \\
\hline$\overleftrightarrow{T} R_{11 k}$ (Municipality 1 ) & {$[12.1,16.1]$} & {$[13.3,17.7]$} & {$[14.6,19.5]$} \\
\hline$\overleftrightarrow{T} R_{12 k}$ (Municipality 2) & {$[10.5,14.0]$} & {$[11.6,15.4]$} & {$[12.8,16.9]$} \\
\hline$\overleftrightarrow{T} R_{13 k}$ (Municipality 3 ) & {$[12.7,17.0]$} & {$[14.0,18.7]$} & {$[15.4,20.6]$} \\
\hline \multicolumn{4}{|c|}{ Cost of waste transportation to WTE facility 1 (\$/tonne): } \\
\hline$\overleftrightarrow{T} R_{21 k}$ (Municipality 1 ) & {$[9.6,12.8]$} & {$[10.6,14.1]$} & {$[11.7,15.5]$} \\
\hline$\overleftrightarrow{T} R_{22 k}$ (Municipality 2) & {$[10.1,13.4]$} & {$[11.1,14.7]$} & {$[12.2,16.2]$} \\
\hline$\vec{T} R_{23 k}$ (Municipality 3 ) & {$[8.8,11.7]$} & {$[9.7,12.8]$} & {$[10.6,14.0]$} \\
\hline \multicolumn{4}{|c|}{ Cost of waste transportation to WTE facility 2 (\$/tonne): } \\
\hline$\overleftrightarrow{T} R_{31 k}$ (Municipality 1$)$ & {$[12.1,16.1]$} & {$[13.3,17.7]$} & {$[14.6,19.5]$} \\
\hline$\overleftrightarrow{T} R_{32 k}$ (Municipality 2) & {$[12.8,17.1]$} & {$[14.1,18.8]$} & {$[15.5,20.7]$} \\
\hline$\overleftrightarrow{T} R_{33 k}$ (Municipality 3 ) & {$[4.2,5.6]$} & {$[4.6,6.2]$} & {$[5.1,6.8]$} \\
\hline \multicolumn{4}{|l|}{$\begin{array}{l}\text { Cost of residue transportation from the WTE } \\
\text { Facilities to the landfill (\$/tonne): }\end{array}$} \\
\hline$\vec{F} T_{2 k} \quad($ WTE facility 1$)$ & {$[4.7,6.3]$} & {$[5.2,6.9]$} & {$[5.7,7.6]$} \\
\hline$\vec{F} T_{3 k} \quad($ WTE facility 2$)$ & {$[13.4,17.9]$} & {$[14.7,19.7]$} & {$[16.2,21.7]$} \\
\hline \multicolumn{4}{|l|}{ Operational cost (\$/tonne): } \\
\hline$\vec{O} P_{1 k} \quad$ (Landfill) & {$[30,45]$} & {$[40,60]$} & {$[50,80]$} \\
\hline$\vec{O} P_{2 k} \quad($ WTE facility 1$)$ & {$[55,75]$} & {$[60,85]$} & {$[65,95]$} \\
\hline$\vec{O} P_{3 k} \quad$ (WTE facility 2) & {$[50,70]$} & {$[60,80]$} & {$[65,85]$} \\
\hline
\end{tabular}


$1,2,3$, corresponds to the time period and $m, m=1,2,3$, 4 , denotes the expansion option selected for the WTE facilities. The decision variables for the problem will be designated by $x_{i j k}, y_{k}$ and $z_{i m k}$, where $x_{i j k}$ represents the proportion of solid waste sent from municipality $j$ to waste processing facility $i$ in period $k ; y_{k}$ corresponds to a binary decision variable for landfill expansion at the start of time period $k\left(y_{k}=1\right.$ if the landfill expands in period $\mathrm{k}, 0$ otherwise), and $z_{\text {imk }}$ represents a binary decision variable corresponding to the particular expansion option, $m$, selected for WTE facility $i, i=2,3$, at the start of period $k$.

The total cost of waste management, in \$/tonne, for the waste flowing from municipality $j$ to processing facility $i$ in period $k$ is represented by $\mathscr{C}_{i j k}$. The per tonne cost for transporting waste from municipality $j$ to facility $i$ in period $k$ is $\vec{T}_{i j k}$, and $\vec{O} P_{i k}$ is the per tonne operating cost of processing facility $i$ in period $k$. The transportation cost per tonne of waste from WTE facility $i$ to the landfill in period $k$ is denoted by $\vec{F} T_{i k}$, for $i=2,3$. If $F E$ represents the residue flow rate from a WTE facility to the landfill, expressed as a percentage of the incoming mass to the WTE facility, and $\vec{R} E_{k}$ denotes the per tonne revenue from the WTE facilities in period $k$, then $\vec{C}_{1 j k}=$ $\vec{T} R_{1 j k}+\vec{O} P_{1 k}$ and $\vec{C}_{i j k}=\vec{T} R_{i j k}+\vec{O} P_{i k}+F E\left(\vec{F} T_{i k}+\right.$ $\left.\vec{O} P_{i k}\right)-\vec{R} E_{k}$, for $i=2,3, j=1,2,3$, and $k=1,2,3$. The existing capacity of the landfill, in tonnes, is $\vec{L} C$ and $\vec{\Delta} L C_{k}$ represents the additional capacity resulting from the landfill expansion undertaken in period $k, k=1,2,3$; where the cost of this landfill expansion is $\vec{F} L C_{k}$. The existing capacity, in tonnes per day, for WTE facility $i, i=$ 2,3 is $\vec{T} C_{i}$. The amount of incremental capacity expansion, in tonnes per day, under option $m, m=1,2,3,4$, for WTE facility $i, i=2,3$, at the start of period $k, k=1,2,3$, is provided by $\vec{\Delta} T C_{i m k}$; with $\vec{F} T C_{i m k}$ corresponding to the capital cost of this expansion. Finally, if the number of days in time period $k$ is $L_{k}$ and if the number of tonnes of waste generated daily by municipality $j$ during period $k$ is $\vec{W} G_{j k}$, then the complete mathematical model for MSW management planning is to:

Minimize

Cost $=\sum_{k=1}^{3} \vec{F} L C_{k} y_{k}+\sum_{i=2}^{3} \sum_{m=1}^{4} \sum_{k=1}^{3} \vec{F} T C_{i m k} z_{i m k}+$

$\sum_{i=1}^{3} \sum_{j=1}^{3} \sum_{k=1}^{3} L_{k} \vec{C}_{i j k} \vec{W}_{j k} x_{i j k}$

Subject to:

$$
\begin{aligned}
& \sum_{j=1}^{3} \sum_{k=1}^{k^{\prime}} L_{k}\left[\vec{W} G_{j k} x_{1 j k}+\sum_{i=2}^{3} \vec{W} G_{j k} x_{i j k} F E\right] \\
& \leq \sum_{k=1}^{k^{\prime}} \vec{\Delta} L C_{k} y_{k}+\vec{L} C
\end{aligned}
$$

$k^{\prime}=1,2,3$ [landfill capacity constraints]

$\sum_{j=1}^{3} \vec{W} G_{j k^{\prime}} x_{i j k^{\prime}} \leq \sum_{m=1}^{4} \sum_{k=1}^{k^{\prime}} \vec{\Delta} T C_{i m k} z_{i m k}+\vec{T} C_{i}$

$i=2,3, \quad k^{\prime}=1,2,3$

[WTE facility capacity constraints]

$$
\sum_{i=1}^{3} \vec{W} G_{j k} x_{i j k} \leq \vec{W} G_{j k}
$$$$
k=1,2,3, \quad j=1,2,3
$$

[waste disposal demand constraints]

$$
\begin{aligned}
& \sum_{m=1}^{4} z_{i m k} \leq 1 \\
& i=2,3, \quad k=1,2,3
\end{aligned}
$$

[only one WTE facility expansion may occur in any given time period]

$$
\sum_{k=1}^{3} y_{k} \leq 1
$$

[landfill expansion may only be considered once]

$\sum_{i=1}^{3} x_{i j k}=1$

$j=1,2,3, \quad k=1,2,3$

[all municipal waste generated must be disposed]

$1 \geq x_{i j k} \geq 0$

$i=1,2,3, \quad j=1,2,3, \quad k=1,2,3$

[non-negativity and proportion constraints]

$y_{k}=0$ or 1

$k=1,2,3$

[binary landfill expansion constraints]

$z_{\text {imk }}=0$ or 1

$i=2,3, \quad k=1,2,3, \quad m=1,2,3,4$

[binary WTE facility expansion option constraints]

Objective function (1) is comprised of components relating to the capital costs required for expanding the processing facilities and the cost/benefit effects resulting from different waste management decisions. Constraints (2) and (3) ensure that the upper limits for waste treatment and disposal in any time period are determined by both the existing facility capacity and any incremental expansion 
activities for the landfill and WTE facilities. The dynamic aspects of these constraints result from future economic development, population increase, and environmental management activities. Constraint (4) establishes the waste disposal quantities generated by each of the three municipalities in each time period. Constraint (5) requires that only one option for each WTE facility expansion can be selected in any given time period, and constraint (6) stipulates that the landfill could be expanded only once over the entire planning time horizon. Constraint (7) ensures the disposal of all waste generated by each municipality in every time period. Finally, constraints (8) to (10) provide the technical relationships for the decision variables.

Since the majority of the MSW system data contains uncertainties than can be estimated only by intervals, Huang et al. (1996b) solved this problem using the GHSJ approach. The optimal solution to the model outlined above provided a grey objective function value, expressed as an interval of $[385.8,690.9]$ million dollars. As indicated earlier, MSW planners faced with difficult and controversial expansion choices would generally prefer to be able to choose from a set of near-optimal alternatives that differ from each other in terms of the system structure characterized by their decision variables. In order to create these alternative planning options, Huang et al. (1996b) added extra constraints to the original model which forced the generation of solutions that were different from the initial optimal solution. Three alternative expansion options were created through the inclusion of a technical constraint on the objective function that increased the total system cost of the original model by $2 \%, 5 \%$, and $8 \%$, respectively. Therefore, three alternative MSW system expansion alternatives were created with total system costs for their respective grey objective function values of [393.5, 704.7], [405.1, 725.4], and [416.7, 746.2]. Hence, by adding specific constraints to the original model, the GHSJ method created specific alternative solutions for MSW expansion planning under uncertainty. However, as demonstrated, the MSW planner would create only a finite subset of policy options using GHSJ and many preferable system alternatives could be inadvertently bypassed altogether.

\section{Evolutionary Simulation-Optimization for Generating Solution Alternatives}

Yeomans (2002) provided an evolutionary simulation optimization mechanism for automatically generating many desirable policy options that might be overlooked by an MGA procedure such as GHSJ. This method can create multiple solution options while simultaneously incorporating inherent system uncertainty directly within the alternative generation process. In this section, the evolutionary simulation-optimization approach for generating policy alternatives to problems containing considerable uncertainty will be described and it will be shown how GHSJ can be incorporated within it to en- hance the resulting performance of both methods. More extensive details on efficient computational representations and implementations for these procedures can be found in the original research of Teleb and Azadivar (1994), Pierreval and Tautou (1997), Azadivar and Tompkins (1999), and Yeomans (2001).

Consider a problem in which there are $n$ decision variables, $\boldsymbol{X}_{i}$, expressed as a vector $\boldsymbol{X}=\left[\boldsymbol{X}_{1}, \boldsymbol{X}_{2}, \ldots, \boldsymbol{X}_{n}\right]$. If $D$ corresponds to the problem's feasible region and $F$ represents the objective function, then an optimization problem, P1, can be stated mathematically as:

$\begin{array}{ll}\text { Minimize/Maximize } & F(\boldsymbol{X}) \\ \text { Subject to: } & \boldsymbol{X} \in D\end{array}$

If the objective, $F$, was a single-criteria, deterministic function and $D$ consisted of a set of $p$ deterministic constraints in the form $g_{j}(\boldsymbol{X}) \leq 0, j=1, \ldots, p$, then $\mathrm{P} 1$ could be solved using an optimization technique from one of the fields of linear, non-linear, and integer programming. Procedures such as simulated annealing, tabu search, lagrangian relaxation, neural networks and genetic algorithms might be required for more mathematically intractable formulations of P1 (Reeves, 1993). Regardless of the optimization method selected, when comparing solutions $\boldsymbol{X} \boldsymbol{1}$ and $\boldsymbol{X} \mathbf{2}$ (i.e. two different settings for $\boldsymbol{X}$ ), $\boldsymbol{X} \boldsymbol{1}$ would be a better solution than $X \mathbf{2}$ if it provided a superior value when measured by objective criterion $F$.

However, in modeling complex systems, precise analytical formulations might be unavailable or could be composed of many highly non-linear and stochastic components. Therefore, suppose that some or all of the deterministic parameters in P1 have been replaced by stochastic functions. Such an instance might occur when uncertain parameters within the objective function or constraints have been represented by some form of probability distribution. Another alternative might occur when $r$ constraints of the form $h_{j}(\boldsymbol{X}) \leq 0, j=1, \ldots, r$, need not be satisfied all of the time. If $\omega_{j}$ represents the proportion of the time, or probability, that constraint $j$ may be violated, then such constraints could be expressed in the form $P\left[h_{j}(\boldsymbol{X}) \geq 0\right] \leq \omega_{j}, \quad j=1, \ldots, r$. Such stochastic constraints pose major solution difficulties for optimization problems, since the resulting systems possess fuzzy feasible region boundaries. P1 can be transformed into a more complicated, multiobjective problem if $F$ were expressed as a vector-valued function consisting of $q$ functions $\boldsymbol{F}_{k}, \mathrm{k}=$ $1, \ldots, q$ (Teleb and Azadivar, 1994), or if the feasible region $D$ simultaneously contained decision options from the real, integer and qualitative domains (Pierreval and Tautou, 1997).

Under stochastic conditions, the constraints and objective can only be efficiently estimated by responses obtained through computer simulation. The comparison of any two solutions to P1 would necessitate the evaluation of some statistic of $F$ when $\boldsymbol{X} \boldsymbol{1}$ is modelled to the same statistic when $\boldsymbol{X} \mathbf{2}$ is modelled (Pierreval and Tautou, 1997). Necessarily, these statistics would be generated by a simulation analysis performed on each solution considered. 
Hence, computer simulation provides the only effective means for comparing results in complex systems. However, simulation in no way provides the means for determining the optimal solution, $\boldsymbol{X}^{*}$, that can be demonstrably proven to possess the best objective function value, $F\left(X^{*}\right)$. Therefore, simulation, by itself, cannot be used as the optimization method for stochastic instances of problems such as $\mathrm{P} 1$.

Hence, irrespective of the actual optimization approach adopted, the selected method would need to undertake some form of search process through the feasible region, $D$. Since the measures of system performance would be stochastic, each potential solution, $\boldsymbol{X}$, would necessarily be evaluated via simulation; with the candidate solution providing the settings for the decision variables under which each simulation would be run. For each candidate solution, the performance measure, $F$, would be estimated by a function of responses generated in the simulation. In practice, stochastic versions of $\mathrm{P} 1$ have commonly been "optimized" either by trial-and-error or by complete enumeration (Azadivar and Tompkins, 1999). Trial-and-error essentially corresponds to a haphazard random search process, while enumeration becomes completely impracticable for anything other than trivially small-sized problems. Other than complete enumeration, the most commonly prescribed optimization methods for directing the solution search have been simulated annealing (Reynolds and McKeown, 1999) and evolutionary algorithms (Azadivar and Tompkins, 1999; Fontanili et al., 2000; Pierreval and Tautou, 1997; Huang et al., 2001).

Typically a simulated annealing algorithm progresses from one candidate solution to another in an attempt to find an improved solution and, hence, this search process examines only a single portion of the feasible region at a time. In contrast, a stochastic search strategy directed by an evolutionary procedure maintains a set (or population) of current candidate solutions throughout its search process, and advances from an entire current population to an entire subsequent population. Each candidate solution's performance (or fitness) is measured by an evaluation of its criterion $F$. The entire population of solutions is considered during each step (or generation) of the evolutionary search and the fitness of each candidate solution is ranked in comparison to every other candidate solution in the current population. Evolutionary search algorithms are probabilistic in nature and, since they are based upon a population of solutions, permit a simultaneous exploration of several regions within $D$. The probabilistic nature of the search occurs in the construction process undertaken for new candidate solutions. Each new population is created from the current generation's population using a selection rule based upon the fitness values obtained for each existing solution. One of the essential principles of evolutionary algorithms is that stronger solutions in the current candidate population should possess a greater likelihood for survival and progression into the next generation. This principle can be established by having the probability of an individual member from the current generation being selected for progression to the subsequent generation proportional to its current fitness measure. Such intrinsic principles render evolutionary search algorithms much less sensitive to local extrema than other methods (Goldberg, 1989).

Evolutionary simulation-optimization procedures commence from a set of candidate solutions stored in an initial population. The process consists of two distinct phases: an evolutionary optimization search phase and a simulation phase. Each candidate solution from the current population is evaluated in the simulation module. The quality of a solution (its fitness for survival) is determined by evaluating its performance criterion, $F$, using simulation analysis. After simulating each candidate solution, the fitness values of each solution are returned to the evolutionary component to be utilized in the creation of the next generation of candidate solutions. These fitness measures become inputs for the evolutionary phase where the next population is generated through the designated evolutionary method for the creation of new candidate solutions. The evolutionary optimization algorithm automatically evolves the system toward improved solutions in subsequent populations and ensures that the search process does not become fixated at some local optima. Upon the creation of the new set of candidate solutions, the new population is returned to the simulation phase for evaluation. This two-phase search process terminates when some appropriately stable system state has been achieved and several methods for accelerating this convergence have been suggested in the literature (Azadivar and Tompkins, 1999; Pierreval and Tautou, 1997). The best solution found during the search will be the optimal solution produced by the procedure for $\mathrm{P} 1$.

Since public policy problems of any consequence would be characterized by large complex systems possessing many uncertain elements, any realistic mathematical model of them would necessarily require a stochastic version of P1 (perhaps with multiple objectives and multiple variable types). In such a representation, system uncertainty would be directly incorporated through the inclusion of stochastic parameter estimation and by permitting certain specific constraint violations for a reasonable proportion of the time. In previously published studies, evolutionary simulation-optimization had only been used for function optimizing purposes (Azadivar and Tompkins, 1999; Fontanili et al., 2000; Pierreval and Tautou, 1997; Huang et al., 2001). Yeomans (2002) extended the method beyond its traditional optimization task into a new role as the generator of policy alternatives for problems containing considerable sources of uncertainty. This novel evolutionary approach provided a mechanism for automatically generating many desirable policy options that would not have been considered otherwise, while simultaneously integrating the inherent planning uncertainty directly into the generation of these alternatives. A synopsis of this evolutionary MGA extension follows. 
Due to its inherent characteristics, an evolutionary algorithm maintains a set of several solutions throughout its searching phase (its population), with the search progressing from one population of candidate solutions to the next. Each solution retained in the population would correspond to one particular policy alternative and, therefore, the population of these candidate solutions would represent a set of policy options for the problem under study. Regardless of the exact evolutionary search procedure employed, during the progression from one generation to a subsequent one, relatively poorer candidate solutions in the population (as measured by objective criterion, $F$ ) would progressively become replaced by better ones in an evolutionary "survival-of-the-fittest" analogy (Holland, 1992; Goldberg, 1989, 1991; Caudill and Butler, 1990). The comparative quality for ranking these solutions would be established by the statistic calculated for each of their respective objectives in the simulation phase of the simulation-optimization procedure. The surviving solutions from the evolutionary actions of the optimization phase would be retained on a "survival of the fittest" basis using this statistical measure. Thus, when the optimization search procedure of $\mathrm{P} 1$ concludes, the final population would necessarily be representative of a highly "fit" population. Therefore, when the evolutionary simulation-optimization algorithm terminates, it would not only have found the single best answer from its solution search, but also have created a set of "near-best" solutions residing in this terminal population (Dibble and Densham, 1993). Therefore, in actuality, evolutionary simulation-optimization has efficiently generated a family of several good solution alternatives for the problem studied. It is this final, "fit" population that corresponds to the set of desirable alternatives to be considered for implementation by policy planners.

Hence, evolutionary simulation-optimization processes can be used to automatically generate a set of desirable alternative policy options. By adopting this approach, multiple policy alternatives can be created that meet the established system criteria, while simultaneously remaining acceptable and implementable in practice. These solutions can be viewed as desirable from two perspectives. Primarily, based upon the evolving nature of the search process employed, all of the surviving solutions will be extremely "fit", corresponding to policy solutions that have necessarily achieved close-to-optimal measures for their objective function value(s). Secondly, the solutions found will all satisfy the stated system requirements that each policy option must possess, as represented by the constraints applied within the problem. This characteristic would result from the evolutionary procedure discarding elements that do not satisfy the necessary "traits" required by members of its population. [Note that because of the stochastic aspects introduced in these problems, some constraints need not be strictly satisfied $100 \%$ of the time].

Due to the existence of many possible solutions to large stochastic system problems expressed in the form of $\mathrm{P} 1$, the solution quality found for such problems can be highly variable unless a thorough search throughout the feasible region has been performed. Evolutionary techniques provide such thorough searches because the disparate candidate solutions maintained in their population permit a concurrent search through multiple portions of the feasible domain. However, since an evolutionary search procedure is a probabilistic process, the actual solution time is stochastic. Therefore, in performing an extensive search for the optimal solution, search times can vary considerably from one run on a specific problem instance to the next (or equivalently, over a fixed period of search time, the solution quality could be highly variable). Hence, one major difficulty experienced by any evolutionary simulation-optimization procedure has been the length of time required for it to converge to an optimal solution (Lacksonen, 2001). Logically, this difficulty also extends to their effectiveness for generating solution alternatives. In the search process, evolutionary procedures evolve from one population to a subsequent one. Consequently, an important aspect to evolutionary simulation-optimization methods is the determination of the initial population of candidate solutions from which the search procedure commences. In general, for a variety of reasons, initial populations for evolutionary searches have been randomly generated (Caudill and Butler, 1990; Goldberg, 1989, 1991; Holland, 1992). However, Reeves (1993) suggested that a directed generation of the initial population using some other means may prove more efficient in speeding up solution convergence. If some computationally efficient method can be used to provide the means for generating a good initial population, then this population may be used to quickly direct the search into preferred regions of a potentially large feasible domain. This initial population biasing can reduce the sole reliance on the evolutionary aspects of the search procedure. Such a directed search may be used to significantly reduce the solution time while simultaneously increasing the solution quality.

Since there is already a dearth of techniques available for solving complex problems containing significant uncertainty, finding this efficient "other means" is not a straightforward process. In practice, had the planner already possessed an efficient solution technique, then, rather than employing it as a stepping stone to some other procedure, this method would more than likely be directly employed for satisfactorily solving the problem. However, it has been described how Huang et al. (1996b) used GHSJ to generate alternatives for complex problems containing uncertainty. Thus, this efficient technique does exist for quickly generating good initial possible populations from which to start an evolutionary search. In the next section, the effectiveness of directing the start of an evolutionary simulation-optimization procedure based upon solutions created by GHSJ will be demonstrated for an MSW case study problem (Huang et al., 1996b). 


\section{Application to the MSW Expansion Planning Case}

Any decision-making procedure employed to support planners in setting public policy must be robust enough to balance the impact of many contradictory and competing factors, while retaining sufficient flexibility to process multiple uncertain sources inherent within the system. Since MSW systems exhibit conflicting characteristics often associated with public policy planning issues, they provide an appropriately representative vehicle for testing any MGA method that can incorporate uncertainty. In this section, the usefulness in combining GHSJ with the evolutionary approach will be illustrated through its application to the case for planning MSW system expansion introduced earlier.

It had been noted that in the GHSJ study, all uncertain inputs and outputs had been expressed as fluctuation intervals. Thus, GHSJ does not require any probability distribution assumptions. This provides an advantage for any "real life" implementation of GHSJ, since it is generally quite difficult for practitioners to specify appropriate distributions that capture their intrinsic system uncertainty. However, the stochastic versions of P1 necessary for evolutionary simulation-optimization require that each uncertain parameter be estimated by some distribution. This distribution requirement does not prove unduly restrictive, since a straightforward transformation process can be applied to all grey parameters in the case. If any data parameter $A$ is estimated to lie within the interval [a, $b]$, then a standard Bayesian argument can be used that converts this unknown parameter into a randomly generated value from a uniform probability distribution with minimum value $a$ and maximum value $b$ (Grey, 1995; Vaughan Jones, 1991). Therefore, in any mathematical instance of the case, the values for all grey parameters, $\vec{A}$, will be randomly generated from uniform distributions using the range endpoints previously established in the GHSJ study. Should the "true", but unknown, distribution for an uncertain element prove to possess a more central tendency within its expressed interval, then the resulting statistical measures calculated will actually overestimate the true variance of the measure. Thus, this Bayesian-uniform distribution transformation can be considered as a conservative assumption. Additionally, this stochastic aspect permits the simultaneous relaxation of several "hard" constraints. Recognizing the practical implications of this relaxation (Foulds and Thachenkary, 2001), several constraints shown in the model can be formulated as percentiles that allow for their conditions to be violated a certain proportion of the time. Hence, by implementing these changes, the GHSJ case model can easily be reformulated for solution by evolutionary simulation-optimization.

A limitation of earlier MGA methodologies had been their inability to include system uncertainty directly into their generated policy options. The reformulated MSW model directly incorporates these numerous uncertain elements, thereby capturing the innate stochastic dynamics existing within the system. Solving the problem would constitute running the simulation-optimization procedure on a stochastic version of the problem P1 to determine the minimum system cost. Any feasible solution to the model would correspond to one particular policy option for MSW expansion planning. Therefore, determining a specific policy for the system necessitates a formulated solution satisfying constraints (2) to (10), with its cost being evaluated by objective (1). Since a simulation is performed upon each decision variable setting, the stochastic aspects of the cost objective could be measured in several different ways. For experimental purposes, two different system objectives will be optimized: one evaluating the mean cost and the other measuring the maximum possible cost.

Each of these objectives possesses distinct practical benefits and provides meaningful insights for a municipality. Minimizing the mean would produce solutions that maintain low system costs on the average and, therefore, would be quite practical when considered over the long term. However, since municipal budgets provide fixed annual dollar amounts to cover their various programs, municipalities also tend to be extremely risk averse and aim to avoid situations that lead to overspending the budgeted allocations. Recognizing that the total system cost follows some stochastic distribution, a strategy that minimizes the maximum possible cost could, in fact, be far different from one that minimizes the mean cost. Hence, determining solutions which minimize maximum possible costs would satisfy the risk aversion characteristics of municipalities. These solutions might contain relatively high costs on average, but would guarantee that spending would never exceed the maximum objective amount found. Therefore, solving a maximum objective problem could hold distinct advantages in establishing budgets for municipalities that must fund their programs solely through taxation.

Eight separate policy generation experiments for the MSW expansion case were performed using the evolutionary simulation-optimization procedure. The initial population for each evolutionary search was established by the four GHSJ solution alternatives previously found by Huang et al. (1996b). These four starting points represent the overall optimal solution for the model, together with the $2 \%, 5 \%$, and $8 \%$ solution alternatives created when constraints were added during the alternative generation phase of GHSJ. Each experimental problem corresponded to a specific combination of the two objective functions and the four different starting populations. In the experimentation, the evolutionary procedure was run with a fixed population size of 50 for a period of 90 minutes. As noted, for policy design using evolutionary simulation-optimization, the planning focus becomes the final population found after running the procedure. Upon termination, this entire surviving population represents a set of policy options generated for MSW expansion in the municipality. The planners would subsequently need to 
closely evaluate trade-offs among options remaining in the final population and perform a comparative analysis on these options in order to select a desired policy decision for MSW expansion.

Figures 2 to 9 reveal the results generated from the eight computational experiments using the evolutionary procedure. Each figure illustrates the calculated objective values for the 50 policy options residing in the terminal populations. Figures 2 to 5 show the final populations created for the mean cost objective when commencing the solution search from each of the GHSJ alternatives. Figures 6 to 9 provide comparable output for the maximum objective. An analysis of the figures highlights certain interesting characteristics regarding the starting points of the evolutionary searches. Since each specific initial population produces analogous solution patterns under both objective functions, the figures will be discussed on a pair wise basis.

Figures 2 and 6 show very similar system costs for all of the options generated in a search starting from the optimal GHSJ solution. This similarity is exemplified by the very "flat" appearance of the histograms. A detailed examination of the decision variables indicated that these options all tend to suggest quite similar MSW system expansion structures. In fact, the differences between the alternatives occur mainly in the proportion allocation variables, $\boldsymbol{x}_{i j k}$, used to direct waste flow from each municipality to the processing facilities. Hence, although all of these options are very good from a cost perspective, the actual expansion alternatives are all very similar in terms of the system structure suggested by their decision variables.

Figures 3 and 7 show the options produced when starting the search procedure from the $2 \%$ GHSJ alternative. These options exhibit more cost variability than those in Figures 2 and 6, and the lower-bound-cost options produced in those found in the corresponding previous scenarios. In comparison to the previous situation, more variety in system structure also results from this starting scenario. Therefore, if the goal of an MGA approach is to produce solution alternatives that vary significantly from one another, this scenario has indicated that evolutionary MGA procedures produce more desirable results when starting from an initial GHSJ population that is suboptimal. Hence, from system cost and solution variety standpoints, starting the search from the $2 \%$ GHSJ alternative produces more desirable results than those produced by the optimal GHSJ option.

Figures 4 and 8 provide the alternatives created when starting from the 5\% GHSJ solution. As with the $2 \%$ scenario, these figures portray considerably more variability than those illustrated in Figures 2 and 6. However, the lower-bound-cost solutions found are not quite as good as those produced by either of the previous starting scenarios, and the upper-bound-cost solutions are considerably higher. Hence, this indicates that the evolutionary search has demonstrated considerable cost improvement in progressing from its initial population and may have been able to produce a lower-bound-cost solution comparable to the earlier scenarios had longer search periods been permitted. However, the lower-bound-cost solutions indicate very good system cost objectives, and the populations provide a significantly more varied set of policy options for the planners than those found from the previous starting points.

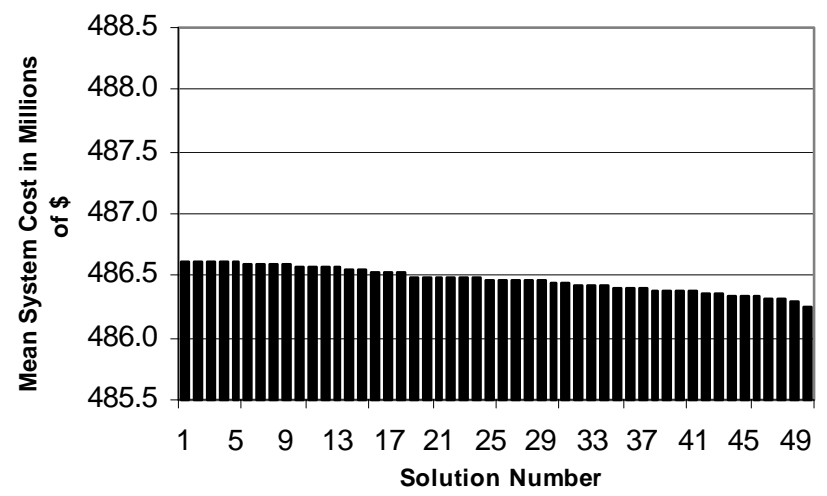

Figure 2. Mean system costs for 50 generated solution alternatives: starting from GHSJ overall optimal solution

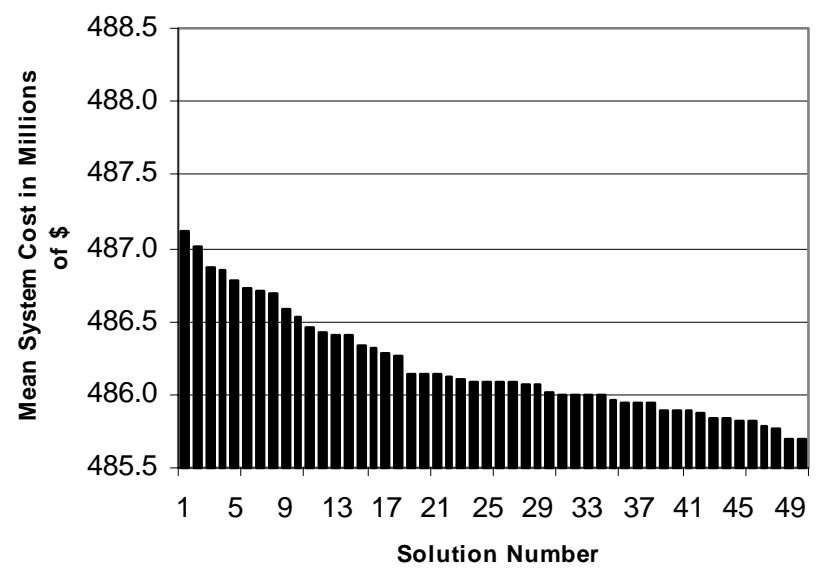

Figure 3. Mean system costs for 50 generated solution alternatives: starting from GHSJ $2 \%$ solution

Finally, Figures 5 and 9 illustrate the policy options generated when starting from the $8 \%$ GHSJ alternative. As with the results from the $5 \%$ starting point, significant solution variability has been produced and the lower-bound-cost solutions are also not quite as good as those found in the first two scenarios. The conclusions applicable to the $5 \%$ starting solution hold equally true for this $8 \%$ solution.

In Figures 2 to 9, all solution option costs cluster at the lower end of the cost ranges that had been produced in the GHSJ study. This result demonstrates that evolutionary simulation-optimization can be used to not only generate considerably more decision options than GHSJ, but also create policy options that guarantee lower costs than those alternatives produced solely by GHSJ. 


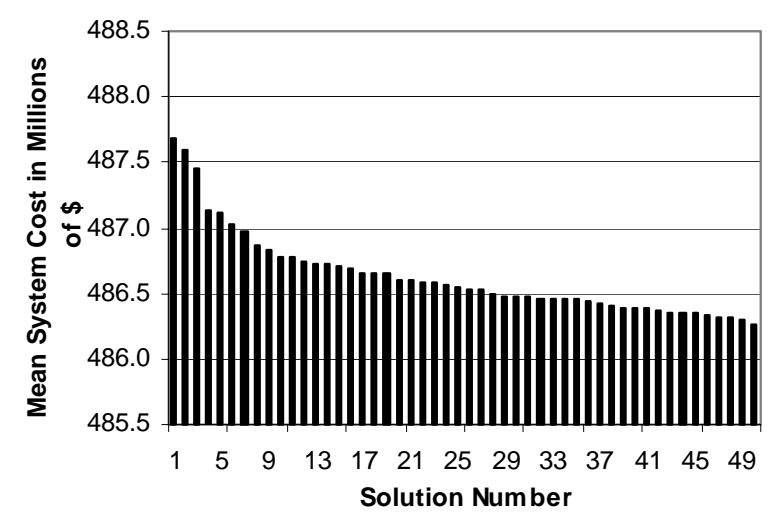

Figure 4. Mean system costs for 50 generated solution alternatives: starting from GHSJ 5\% solution

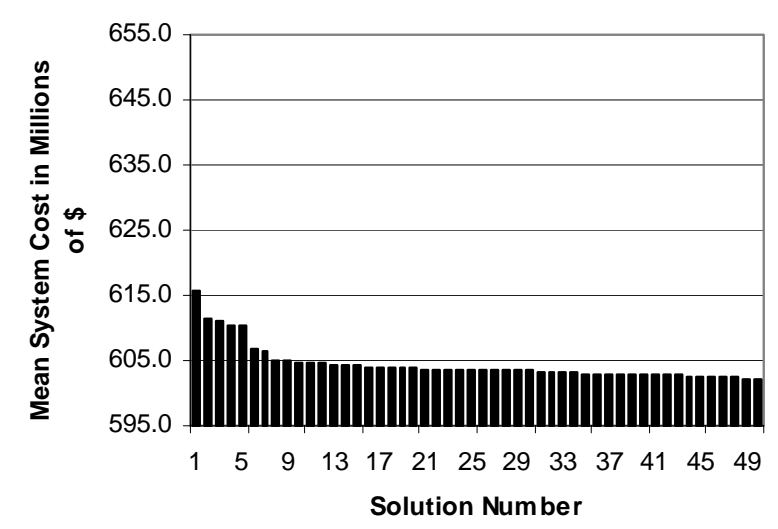

Figure 6. Maximum system costs for 50 generated solution alternatives: starting from GHSJ overall optimal solution

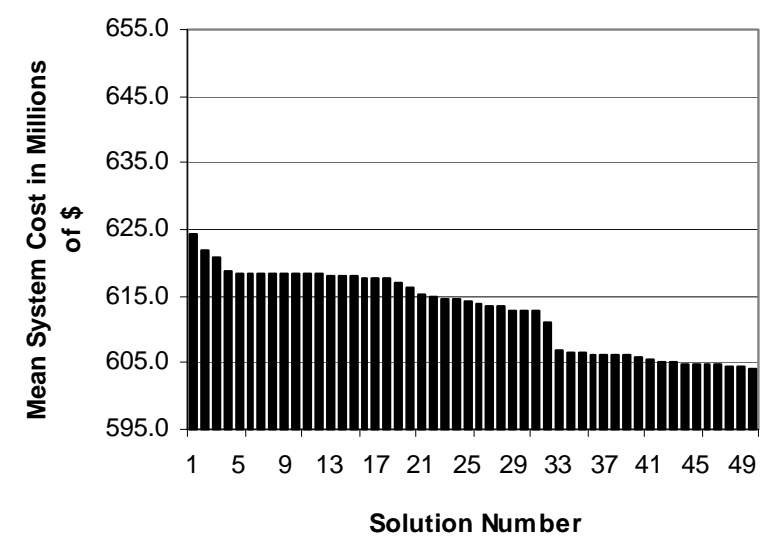

Figure 8. Maximum system costs for 50 generated solution alternatives: starting from GHSJ $5 \%$ solution

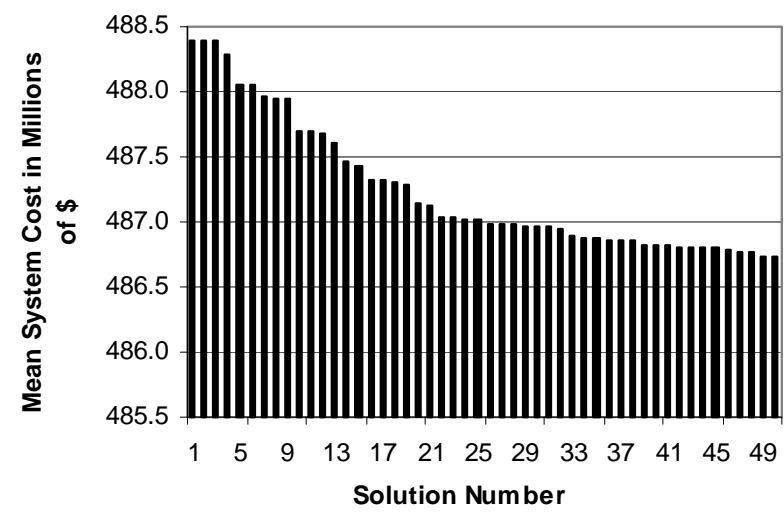

Figure 5. Mean system costs for 50 generated solution alternatives: starting from GHSJ $8 \%$ solution

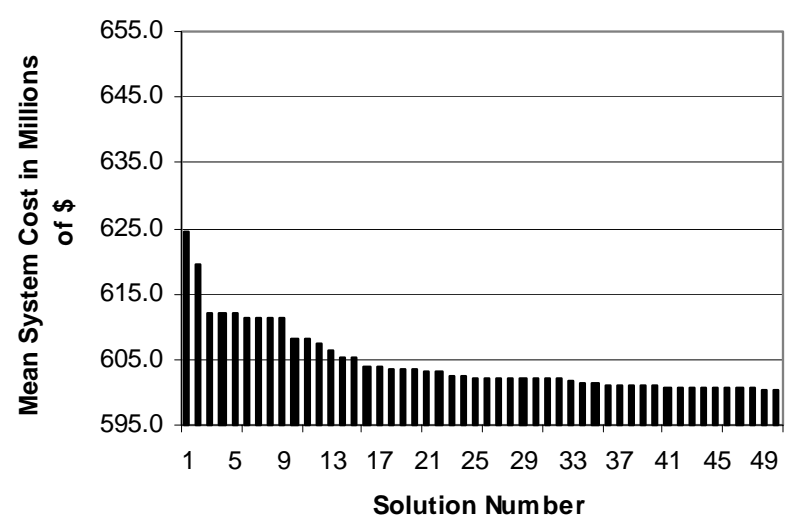

Figure 7. Maximum system costs for 50 generated solution alternatives: starting from GHSJ $2 \%$ solution

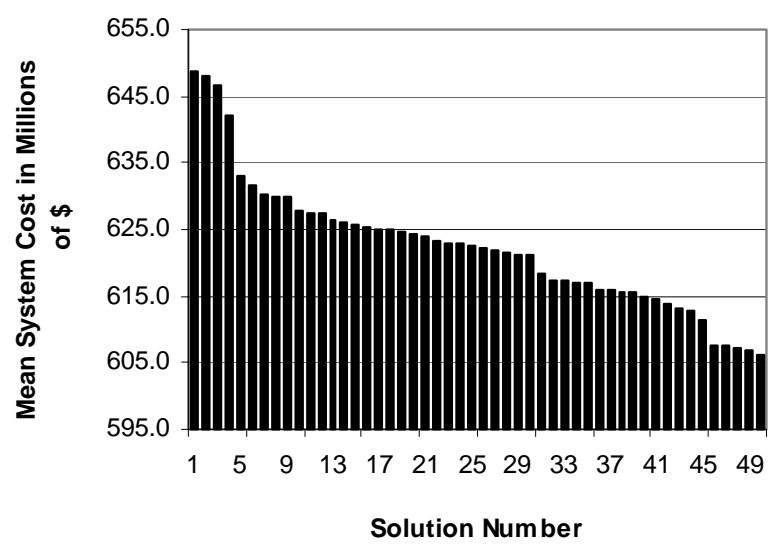

Figure 9. Maximum system costs for 50 generated solution alternatives: starting from GHSJ $8 \%$ solution 
Furthermore, the computational experimentation has demonstrated the apparent utility of starting an evolutionary solution search from one of the suboptimal GHSJ alternatives. Such a less restrictive starting point seems to permit more leeway for the search to evolve toward more desirable solutions, while simultaneously producing structurally diverse policy alternatives. This finding was evident even when starting from the marginally suboptimal, $2 \%$ GHSJ alternative. The final population sets generated from the $5 \%$ and $8 \%$ GHSJ alternatives produced even more variety in the policy options created, and would have quite conceivably produced objective values of comparable quality to the other starting points had additional search time been permitted. Thus, it appears that good practice would dictate that an evolutionary search should start from one of the suboptimal GHSJ alternatives to ensure the production of more solution variety in the policies generated.

To summarize the results in this section, the highly efficient GHSJ procedure has been used to quickly provide several initial starting points for improving both the solution time and performance quality of evolutionary simulation-optimization. The set of multiple policy alternatives generated by the hybrid GHSJ-evolutionary procedure provides an extremely efficient means for incorporating inherent system uncertainty directly into difficult policy construction processes. The case study presented in this section has demonstrated that this proposed hybrid procedure can be successfully exploited to simultaneously expand and improve the policy alternative generation roles of both MGA methods. This procedure has been demonstrated in the design formulation stage for policy option generation and has been shown extremely capable of finding multiple, desirable solid-waste expansion alternatives for the MSW case.

\section{Conclusions}

Major public policy problems are composed of large complex systems complicated by a multitude of uncertain economic, technological, environmental, social, and political dimensions. These conflicting dimensions force planners to consider and integrate many issues prior to the establishment of an acceptable final policy. Hence, it is most unlikely that one best solution can ever be found that simultaneously satisfies the requirements emanating from these dimensions without a significant balancing of the tradeoffs involved. Because inherent system uncertainties and conflicting requirements make it unlikely for a single solution method to capture the entire essence of a situation, MGA approaches have been adopted to create multiple planning alternatives for such policy problems. Since MGA approaches have generally been based upon deterministic procedures, they have not provided an effective means to integrate uncertain information into their resulting solution options. To counteract this deficiency, Huang et al. (1996b) created the GHSJ technique. However, GHSJ can examine only a small subset of possible policy options and, in so doing, could inadvertently bypass a number of preferable system alternatives. Yeomans (2002) provided an evolutionary method that could be used for generating many more solution options than would be created by a GHSJ procedure alone.

In this paper, it was recognized that GHSJ could be used to quickly generate several good starting populations that could then be used to direct the initial stages of an evolutionary search. By combining GHSJ with evolutionary simulation-optimization, the search time and solution quality of both techniques can be improved significantly. Unlike deterministic MGA methods, this combined algorithm permits the automatic construction of multiple solution options that incorporate system uncertainty directly into each generated alternative. The efficacy of the method was demonstrated on an earlier case study application concerning expansion planning in an MSW system. The procedure efficiently produced many solutions possessing the characteristics requisite of the MSW system, with each alternative providing a somewhat different planning perspective. Planners would subsequently review these alternatives and analyze the various solution trade-offs before determining which option to implement as their final policy decision.

In conclusion, this study has demonstrated the practicality of combining an evolutionary simulation optimization procedure with GHSJ for efficiently designing many desirable alternatives for planning problems containing considerable degrees of uncertainty. Although the procedure was demonstrated only upon an MSW case study, it could easily be generalized to many other policy setting situations. An MSW system was selected since these systems exhibit all of the characteristics typically associated with public policy planning. This hybrid technique can clearly be applied to both strategic and operational planning analyses. Future research will extend the application of this approach to other policy planning problems.

Acknowledgments. This research was supported in part by grant OGP0155871 from the Natural Sciences and Engineering Research Council.

\section{References}

Azadivar, F. and Tompkins, G. (1999). Simulation optimization with qualitative variables and structural model changes: A genetic algorithm approach. Eur. J. Oper. Res., 113, 169-182.

Baetz, B.W., Pas, E.I. and Neebe, A.W. (1990). Generating alternative solutions for dynamic programming-based planning problems. Soc.-Econ. Plann. Sci., 24, 27-34.

Bass, B., Huang, G.H. and Russo, J. (1997). Incorporating climate change into risk assessment using grey mathematical programming. J. Environ. Manage., 49, 107-124.

Brill, E.D. (1979). The use of optimization models in public sector planning. Manage. Sci., 25, 413-422.

Brill, E.D., Chang, S.Y. and Hopkins, L.D. (1981). Modeling to generate alternatives: the HSJ approach and an illustration using a problem in land use planning. Manage. Sci., 27, 314-325. 
Caudill, M. and Butler, C. (1990). Naturally Intelligent Systems, MIT Press, Cambridge, MA, USA.

Chang, S.Y., Brill, E.D. and Hopkins, L.D. (1980). Efficient Random Generation of Feasible Alternatives: A Land Use Planning Example, Staff Paper 12, Institute for Environmental Studies, University of Illinois, Urbana, IL, USA.

Chang, S.Y., Brill, E.D. and Hopkins, L.D. (1982). Use of mathematical models to generate alternative solutions to water resources planning problems. Water Resour. Res., 18, 58-64.

Chang, N.B. and Wang, S.F. (1995). A grey nonlinear programming approach for planning coastal wastewater treatment and disposal systems. Water Sci. Technol., 32, 19-29.

Chang, N.B., Wen, C.G. and Yong, Y.C. (1996). A Grey Fuzzy Multiobjective Programming Approach for Optimal Planning of a Reservoir Watershed. Water Res., 30, 2329-2334.

Church, R.L. and Huber, D.L. (1979). On Finding Close to Optimal Solutions as well as Determining a Noninferior Set of Tradeoff Envelopes for Multi-objective Location Models, Research Series 34, Department of Civil Engineering, University of Tennessee, Knoxville, TN, USA

Dibble, C. and Densham, P.J. (1993). Generating interesting alternatives in GIS and SDSS using genetic algorithms, in Proc. of GIS/LIS 1993 American Society for Photogrammetry and Remote Sensing/American Congress on Surveying and Mapping, 5410 Grosvenor Lane, Bethesda, MD, USA.

Falkenhausen, K. (1979). Facility location planning for regional waste treatment systems by branch and bound vs. evolution strategy concepts, in the ORSA/TIMS Milwaukee Conference.

Fontanili, F., Vincent, A. and Ponsonnet, R. (2000). Flow simulation and genetic algorithm as optimization tools. Int. J. Product. Econ., 64, 91-100.

Foulds, L. and Thachenkary, C. (2001). Empower to the people, OR/MS Today, 28(3), 32-37.

Fu, M.C. (1994). Optimization via simulation: A review. Ann. Oper. Res., 53, 199-248.

Gidley, J.S. and Bari, M.F. (1986). Modeling to generate alternatives. Water Forum, 86, 1366-1373.

Goldberg, D.E. (1989). Genetic Algorithms in Search Optimization and Machine Learning, Addison-Wesley, Reading, MA, USA.

Goldberg, D.E. (1991). Genetic Algorithms, Addison-Wesley, Reading, MA, USA

Grey, S. (1995). Practical Risk Assessment, John Wiley and Sons, New York, NY, USA.

Harris, B. (1983). Positive and Normative Aspects of Modeling Large-Scale Social Systems, in M. Batty and B. Hutchinson (Eds.), Systems Analysis in Urban Policy-Making and Planning, Plenum Press, New York, NY, USA, pp. 475-490.

Hasit, Y. and Warner, D.B. (1981). Regional solid waste planning with WRAP. J. Environ. Eng., 107, 511-525.

Haynes, L. (1981). A systems approach to solid waste management planning. Conserv. Recycling, 4(2), 67-78.

Holland, J.H. (1992). Adaptation in Natural and Artificial Systems, 2nd Edition, MIT Press, Cambridge, MA, USA.

Huang, G.H. (1994). Grey Mathematical Programming and Its Application to Waste Management Planning Under Uncertainty, Ph.D. Dissertation, Department of Civil Engineering, McMaster University, Hamilton, Ontario, Canada.

Huang, G.H. (1996). IPWM: An interval parameter water quality management model. Eng. Optimiz., 26, 79-103.

Huang, G.H., Baetz, B.W. and Patry, G.G. (1994a). A grey chance-constrained programming approach for waste management planning under uncertainty, in K.W. Hipel and L. Fang (Eds.), Effective Environmental Management for Sustainable Development, Kluwer Academic Publishers, Dordecht, Netherlands, pp. 267-280.
Huang, G.H., Baetz, B.W. and Patry, G.G. (1994b). Grey dynamic programming for solid waste management planning under uncertainty. J. Urban Plann. Dev., 120, 132-156.

Huang, G.H., Baetz, B.W. and Patry, G.G. (1995). A grey integer programming approach for waste management planning. Eur. $J$. Oper. Res., 83, 594-620.

Huang, G.H., Baetz, B.W. and Patry, G.G. (1996a). A grey hop, skip and jump method for generating decision alternatives: planning for the expansion/utilization of waste Management facilities. Can. J. Civ. Eng., 23, 1207-1219.

Huang, G.H., Cohen, S.J., Yin, Y.Y. and Bass, B. (1996b). Incorporation of inexact integer programming and fuzzy relation analysis for integrated environmental impact assessment and adaptation study under uncertainty. J. Environ. Manage., 48, 45-68.

Huang, G.H., Jorgensen, S.E., Xu, Y. and Guo, H.C. (1997). Integrated Environmental Planning for Sustainable Development in Lake Erhai Basin-with a Diagnostic Study for Local Environmental Concerns, United Nations Environment Programme, Nairobi, Kenya.

Huang, G.H., Baetz, B.W. and Patry, G.G. (1998). Trash-flow allocation: Planning under uncertainty. Interfaces, 28(6), 36-55.

Huang, G.H., Yeomans, J.S. and Yoogalingam, R. (2001). Combining Simulation with Evolutionary Algorithms for Optimal Planning Under Uncertainty: An Application to Municipal Solid Waste Management Planning in the Regional Municipality of Hamilton-Wentworth, Working Paper, Schulich School of Business, York University, Toronto, Ontario, Canada.

Inuiguchi, M., Ichihashi, H. and Tanaka, H. (1990). Fuzzy programming: A survey of recent developments, in $\mathrm{R}$. Slowinski and J. Teghem (Eds.), Stochastic versus Fuzzy Approaches to Mathematical Programming under Uncertainty, Kluwer Academic Publishers, Dordecht, Netherlands, pp. 45-70.

Keeney, R.L. and Raiffa, H. (1976). Decisions with Multiple Objectives: Preferences and Value Tradeoffs, Cambridge University Press, Cambridge, UK.

Kleindorfer, P.R., Kunreuther, H.C. and Schoemaker, P.J.H. (1993). Decision Sciences: An Integrative Perspective, Cambridge University Press, Cambridge, UK.

Lacksonen, T. (2001). Empirical comparison of search algorithms for discrete event simulation. Comput. Ind. Eng., 40, 133-148.

Lund, J.R. (1990). Least cost scheduling of solid waste recycling. J. Environ. Eng., 116, 182-197.

Lund, J.R., Tchobanoglous, G., Anex, R.P. and Lawver, R.A. (1994). Linear programming for analysis of material recovery facilities. J. Environ. Eng., 120, 1082-1094.

Openshaw, B.W. and Whitehead, P. (1975). A decision optimising technique: A rejoinder. Town Plann. Rev., 51, 675.

Pierreval, H. and Tautou, L. (1997). Using evolutionary algorithms and simulation for the optimization of manufacturing systems. IIE Trans., 29(3), 181-189.

Reeves, C.R. (1993). Modern Heuristic Techniques for Combinatorial Problems, John Wiley and Sons, New York, NY, USA.

Rubenstein-Montano, B. and Zandi, I. (1999). Application of a genetic algorithm to policy panning: The case of solid waste. Environ. Plann. B: Plann. Des., 26, 893-907.

Rubenstein-Montano, B., Ananadalingam, G. and Zandi, I. (2000). A genetic algorithm approach to policy design for consequence minimization. Eur. J. Oper. Res., 124, 43-54.

Teleb, R. and Azadivar, F. (1994). A methodology for solving multi-objective simulation-optimization problems. Eur. J. Oper. 
Res., 72, 135-145.

Vaughan Jones, C. (1991). Financial Risk Analysis of Infrastructure Debt, Quorum Books, Westport, CT, USA.

Walker, W.E. (1976). A heuristic adjacent extreme point algorithm for the fixed charge problem. Manage. Sci., 22, 587-596.

Wenger, R.B. and Cruz-Uribe, B.W. (1990). Mathematical mod- els in solid waste management: A survey, in the TIMS/ORSA Las Vegas Conference.

Yeomans, J.S. (2002). Automatic Generation of Efficient Policy Alternatives via Simulation-Optimization, J. Oper. Res. Soc., 53(11), 125-1267 